\section{Calcifying pseudoneoplasm of the neuraxis}

\section{Dear Editor,}

A 17 -year-old male patient with a 12-year history of generalized tonic-clonic seizures was referred to our clinic for diagnostic investigation. Magnetic resonance imaging (MRI) showed an expansile lesion in the left frontal lobe, with a hypointense signal in T1-weighted sequences, a hyperintense signal in T2-weighted sequences, a halo of hypointense signal in both sequences, no contrast enhancement, and mild edema in the surrounding tissue (Figures 1A and 1B). The lesion was completely resected (Figure 1C), after which it was sent for histopathological and immunohistochemical analysis (Figure 1D), which resulted in a diagnosis of calcifying pseudoneoplasm of the neuraxis (CAPNON). At this writing, the patient has been followed for two years since the surgical procedure, with no clinical or radiological signs of recurrence.

A CAPNON, also known as a fibro-osseous lesion of the central nervous system (CNS), is a rare benign lesion, first reported by Rhodes et al. in $1978^{(1)}$. A CAPNON is a slow-growing calcified lesion, usually solitary, which can arise in any part of the CNS. Although its origin is not yet fully known, on the basis of its benign clinical evolution and histopathological findings, it is believed to occur in response to factors such as trauma, infection, inflammation, and even neoplasms ${ }^{(2,3)}$. According to Duke et al. ${ }^{(4)}$, the majority $(59.38 \%)$ of the cases reported in the literature have occurred in men, intracranial (mainly supratentorial) lesions accounting for $54.20 \%$ of all cases. The main symptoms related to this type of lesion are headaches, seizures, and focal neurological symptoms. However, the clinical presentation will depend on the location, size, and consequent mass effect of the lesion $^{(4,5)}$.

Imaging examinations are important tools for the diagnosis of CNS tumors ${ }^{(6-10)}$, also serving to characterize a CAPNON and to include it in the differential diagnosis of calcified lesions of the CNS. On conventional radiography and computed tomography, most CAPNONs reportedly present as solid lesions that are completely calcified or have peripheral calcifications. On MRI, a CAPNON typically shows a hypointense signal in T1- and T2-weighted sequences, with minimal or no contrast enhancement, rarely causing edema ${ }^{(11,12)}$. However, there have been reports of CAPNONs with varying signals in T1- and T2weighted sequences, or even with edema and significant contrast enhancement ${ }^{(12)}$.

The diagnosis of a CAPNON depends on the histopathological study showing nodules with chondromyxoid matrix and fine calcifications, containing psammomatous bodies and varying quantities of fibrous stroma, as well as epithelioid and fusiform cells in the periphery. The presence of each of those components is highly variable among the cases reported. The most common immunohistochemical findings are the presence of epithelial membrane antigen and vimentin, together with the absence of glial fibrillary acid protein and of S-100 protein $^{(3,12)}$.

Surgical resection is the treatment of choice for CAPNON and usually improves the prognosis, there having been no reports of recurrence after complete removal of the lesion ${ }^{(11)}$. Some of the differential imaging diagnoses are cavernoma, meningioma, infectious lesions (neurocysticercosis, tuberculosis),

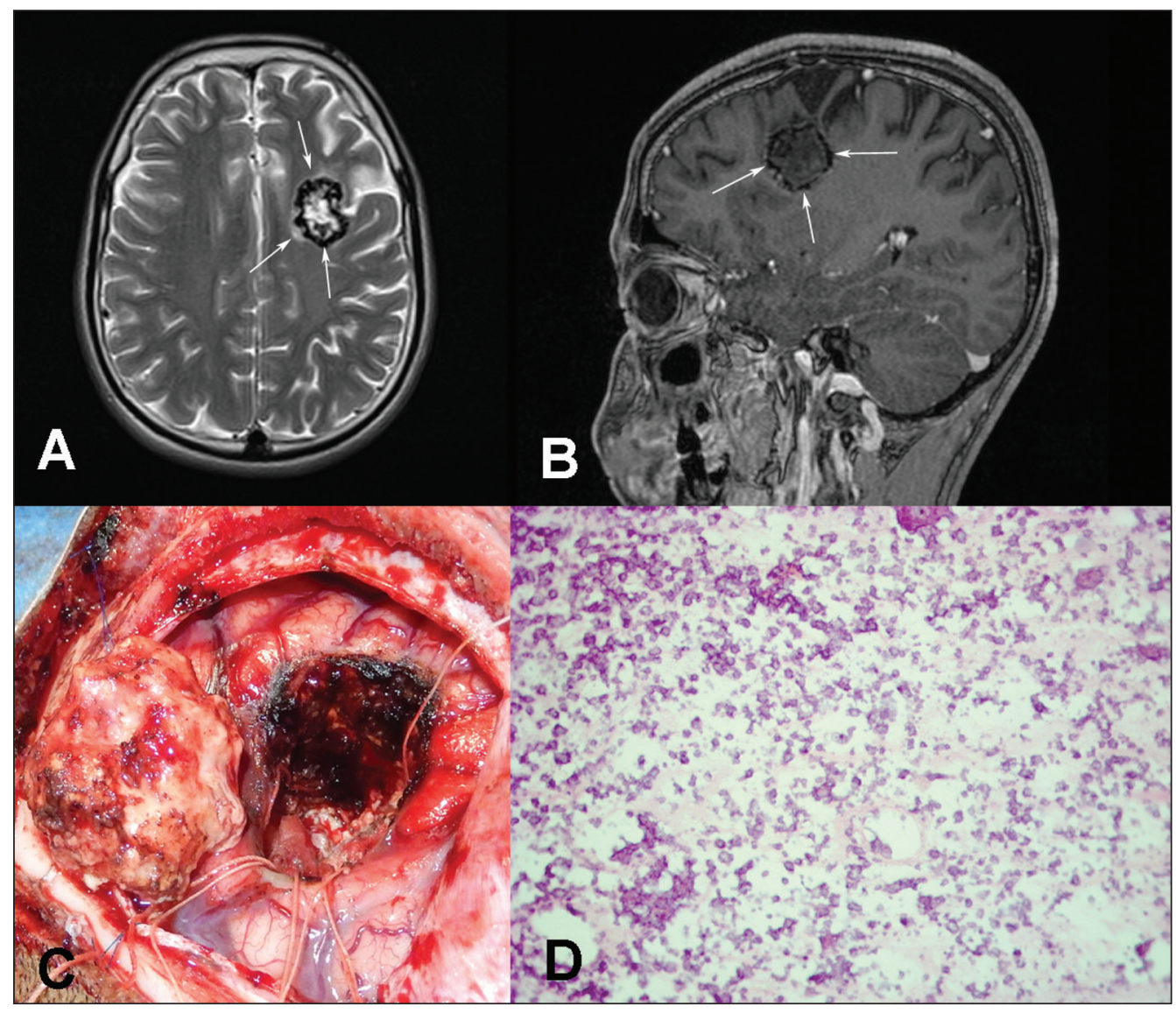

Figure 1. Preoperative MRI. Unenhanced T2-weighted sequence (A) and contrast-enhanced T1-weighted sequence (B) showing an expansile lesion in the left frontal lobe, with a halo of hypointense signal in both sequences, together with an internal component showing a hyperintense signal in T2-weighted sequences and a hypointense signal in T1-weighted sequences, without contrast enhancement and with mild edema in the surrounding tissue. C: Intraoperative image of a CAPNON after total resection. D: Histological slice showing numerous foci of calcification (microcalcifications), corresponding to cells from the lesion. Rare viable cells within the area were identified only in the immunohistochemical study (hematoxylin and eosin, magnification, 20x). 
oligodendroglioma, and astrocytoma with calcifications ${ }^{(3,12,13)}$ Due to the increasing number of cases described in recent years, CAPNON should be included in the differential diagnosis of calcified CNS lesions.

\section{REFERENCES}

1. Rhodes RH, Davis RL. An unusual fibro-osseous component in intracranial lesions. Hum Pathol. 1978;9:309-19.

2. Aiken AH, Akgun H, Tihan T, et al. Calcifying pseudoneoplasms of the neuraxis: CT, MR imaging, and histologic features. AJNR Am J Neuroradiol. 2009;30:1256-60.

3. Serrano-Pardo R, Valls AB, Benítez HS, et al. Pseudoneoplasia calcificante del sistema nervioso central. A propósito de un caso y revisión de la literatura. Rev Esp Patol. 2009;42;155-8.

4. García Duque S, Medina Lopez D, Ortiz de Méndivil A, et al. Calcifying pseudoneoplasms of the neuraxis: report on four cases and review of the literature. Clin Neurol Neurosurg. 2016;143:116-20.

5. Giardina F, Guerra G, Righi A, et al. Calcifying pseudotumor of the spine: a case report. Clin Surg. 2016;1:1042.

6. Queiroz RM, Abud LG, Abud TG, et al. Burkitt-like lymphoma of the brain mimicking an intraventricular colloid cyst. Radiol Bras. 2017;50: 413-4.

7. Langer FW, Santos D, Alves GRT, et al. Malignant peripheral nerve sheath tumor of the vagus nerve: an uncommon cause of progressive dyspnea. Radiol Bras. 2017;50:412-3.

8. Abreu PP, Muniz BC, Ventura N, et al. Intraventricular ganglioglioma with dissemination of cerebrospinal fluid. Radiol Bras. 2018;51:272-3.
9. Niemeyer B, Muniz BC, Ventura N, et al. Papillary tumor of the pineal region accompanied by Parinaud's syndrome: magnetic resonance imaging findings. Radiol Bras. 2018;51:202-4.

10. Muniz BC, Niemeyer B, Ventura N, et al. Primary intraosseous meningioma: atypical presentation of a common tumor. Radiol Bras. 2018;51: 412-3.

11. Krejčí T, Buzrla P, Vecera Z, et al. Calcifying pseudoneoplasm of the neural axis - a case report. Cesk Slov Neurol N. 2015;78/111:568-75.

12. Stienen MN, Abdulazim A, Gautschi OP, et al. Calcifying pseudoneoplasms of the neuraxis (CAPNON): clinical features and therapeutic options. Acta Neurochir (Wien). 2013;155:9-17.

13. Lyapichev K, Bregy A, Shah AH, et al. Occipital calcified pseudoneoplasms of the neuraxis (CAPNON): understanding a rare pathology. BMJ Case Rep. 2014;2014.

Rafaela Fonseca Albuquerque Pithon ${ }^{1, a}$, Paulo Roberto Valle Bahia ${ }^{1, b}$, Jorge Marcondes $^{1, c}$, Nathalie Canedo ${ }^{1, d}$, Edson Marchiori ${ }^{1, e}$

1. Universidade Federal do Rio de Janeiro (UFRJ), Rio de Janeiro, RJ, Brazil. Correspondence: Dra. Rafaela Fonseca Albuquerque Pithon. UFRJ - Departamento de Radiologia. Rua Rodolpho Paulo Rocco, 255, Cidade Universitária, Ilha do Fundão. Rio de Janeiro, RJ, Brazil, 21941-913. Email: rafaelafa@hotmail. com.br.

a. https://orcid.org/0000-0002-8308-1920; b. https://orcid.org/0000-0002-4765-8526; c. https://orcid.org/0000-0003-2412-8239; d. https://orcid.org/0000-0002-9306-7145; e. https://orcid.org/0000-0001-8797-7380.

Received 22 September 2017. Accepted after revision 14 December 2017.

http://dx.doi.org/10.1590/0100-3984.2017.0171

\section{$(c)) \mathrm{Er}$}

\section{Hepatic changes after treatment with oxaliplatin}

\section{Dear Editor,}

A 54-year-old female with colon cancer was treated surgically and with a chemotherapy regimen (fluorouracil, leucovorin, and oxaliplatin). Pre-treatment imaging examinations had shown that her liver had a normal aspect, with no evidence of focal lesions. Follow-up (post-treatment) examinations showed mild splenomegaly and slightly lobulated liver contours. Some liver nodules also appeared, most of them characterized by an isointense signal in T1- and T2-weighted sequences with enhancement in the arterial phase and persistent uptake in the hepatobiliary phase after intravenous injection of a hepatobiliary-specific contrast agent, suggesting focal nodular hyperplasia (FNH)-like lesions (Figure 1).

Figure 1. Magnetic resonance imaging. Unenhanced, fat-suppressed T1-weighted sequence (A), unenhanced T2-weighted sequence (B), and contrast-enhanced T1 weighted sequences in the arterial and hepatobiliary phases (C and $\mathbf{D}$, respectively), showing mild splenomegaly and hepatic nodules with isointense signals on T1- and T2 weighted sequences, arterial enhancement, and persistent enhancement in the hepatobiliary phase, suggesting $\mathrm{FNH}$-like lesions. Other areas containing nodules and showing contrast uptake in the hepatobiliary phase can be seen, also suggesting benignity.

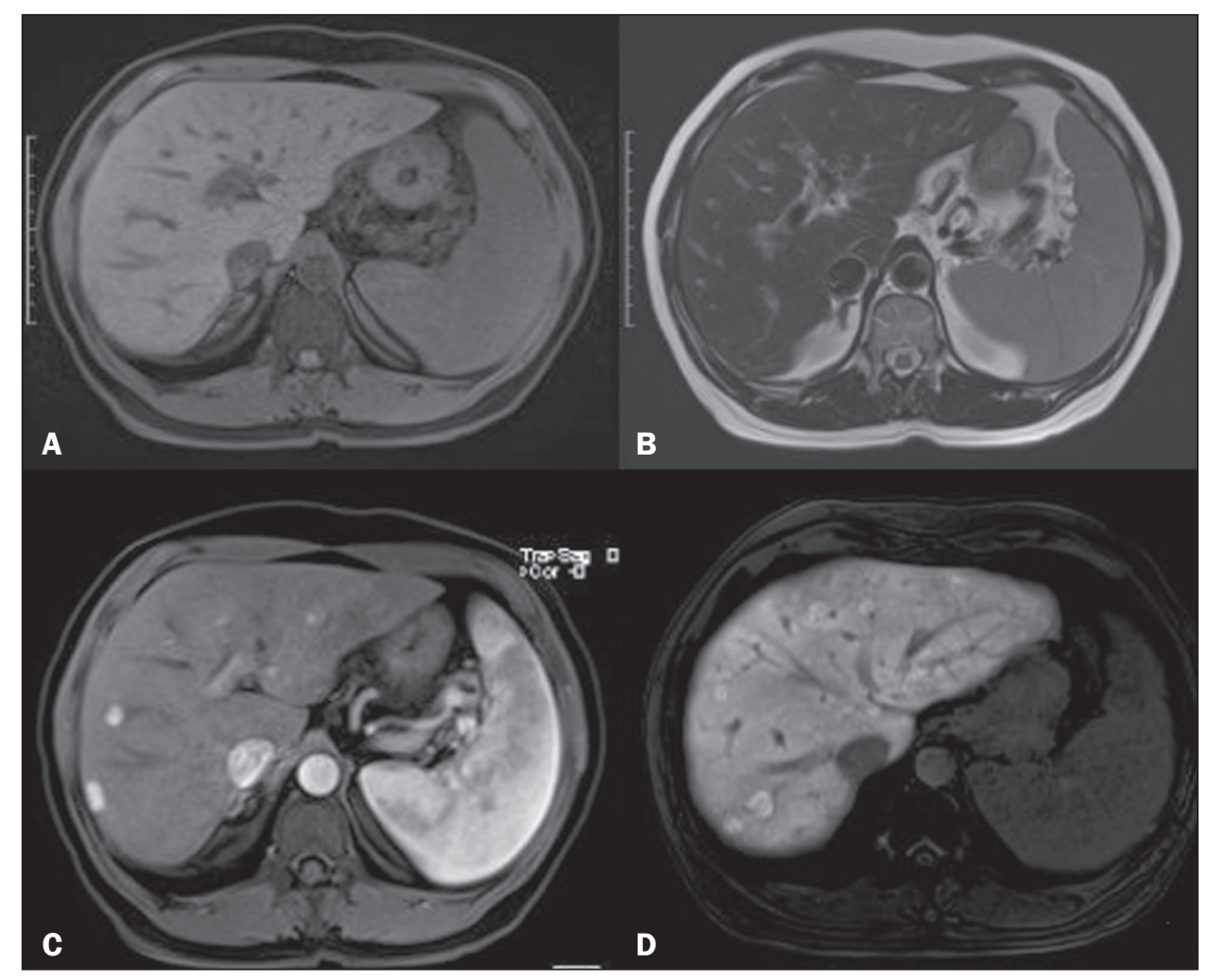

